\title{
BMJ Open \\ “You don't immediately stick a label on them": a qualitative study of influences on general practitioners' recording of anxiety disorders
}

\author{
Elizabeth Ford, ${ }^{1}$ Alice Campion, ${ }^{2}$ Darleen Aixora Chamles, ${ }^{3}$ \\ Haniah Habash-Bailey, ${ }^{1}$ Maxwell Cooper ${ }^{1}$
}

To cite: Ford $E$, Campion $A$, Chamles DA, et al. "You don't immediately stick a label on them": a qualitative study of influences on general practitioners' recording of anxiety disorders. BMJ Open 2016;6:e010746. doi:10.1136/bmjopen-2015010746

- Prepublication history for this paper is available online. To view these files please visit the journal online (http://dx.doi.org/10.1136/ bmjopen-2015-010746).

Received 3 December 2015 Revised 15 March 2016 Accepted 17 March 2016

CrossMark

\footnotetext{
${ }^{1}$ Division of Primary Care and Public Health, Brighton and Sussex Medical School, Brighton, UK

${ }^{2}$ Musgrove Park Hospital, Taunton and Somerset Trust, Taunton, UK

${ }^{3}$ Ysbyty Gwynedd, Betsi Cadwaladr University Health Board (West),

Penrhosgarnedd, Gwynedd, UK
}

\section{Correspondence to} Dr Elizabeth Ford; e.m.ford@bsms.ac.uk

\section{ABSTRACT}

Objectives: Anxiety is a common condition usually managed in general practice (GP) in the UK. GP patient records can be used for epidemiological studies of anxiety as well as clinical audit and service planning. However, it is not clear how general practitioners (GPs) conceptualise, diagnose and document anxiety in these records. We sought to understand these factors through an interview study with GPs.

Setting: UK National Health Service (NHS) General Practice (England and Wales).

Participants: 17 UK GPs.

\section{Primary and secondary outcome measures:}

Semistructured interviews used vignettes to explore the process of diagnosing anxiety in primary care and investigate influences on recording. Interviews were transcribed verbatim and analysed using thematic analysis.

Results: GPs chose 12 different codes for recording anxiety in the 2 vignettes, and reported that history, symptoms and management would be recorded in free text. GPs reported on 4 themes representing influences on recording of anxiety: 'anxiety or a normal response', 'granularity of diagnosis', 'giving patients a label' and 'time as a tool'; and 3 themes about recording in general: 'justifying the choice of code', 'usefulness of coding' and 'practice-specific pressures'. GPs reported using only a regular selection of codes in patient records to help standardise records within the practice and as a time-saving measure.

Conclusions: We have identified a coding culture where GPs feel confident recognising anxiety symptoms; however, due to clinical uncertainty, a longterm perspective and a focus on management, they are reluctant to code firm diagnoses in the initial stages. Researchers using GP patient records should be aware that GPs may prefer free text, symptom codes and other general codes rather than firm diagnostic codes for anxiety.

\section{INTRODUCTION}

Mental health problems represent a large proportion of the disease burden in the UK and are an important cause of long-term

\section{Strengths and limitations of this study}

- An in-depth qualitative study reporting on how general practitioners (GPS) record anxiety and what influences this.

- This is the first study to investigate these issues in anxiety disorders.

- A convenience sample of 17 GPs means that findings cannot be generalised.

- Researchers and policymakers using GP patient records for epidemiological studies should be aware that GPs may prefer descriptive rather than diagnostic codes for anxiety.

disability and dependency. Mental and substance use disorders are the leading cause of 'years lived with disability' (YLDs) worldwide, accounting for $31.7 \%$ of all YLDs. ${ }^{1}$ Anxiety disorders are an important part of this burden, accounting for $14.6 \%$ of disease burden measured in disability-adjusted life years. $^{2}$ Anxiety disorders, such as generalised anxiety disorder (GAD), panic disorder, phobias, obsessive compulsive disorder (OCD) and post-traumatic stress disorder, are common, with a global lifetime prevalence of around $17 \% .{ }^{3}$ In the UK, the point prevalence of anxiety has been reported as follows: mixed anxiety and depressive disorder $9.0 \%$; GAD $4.4 \%$, panic, phobias and OCD $1-1.5 \%{ }^{4}$

Health services are not provided equitably to people with mental disorders. ${ }^{1}$ The WHO calculated the global treatment gap (ie, the percentage of patients who remain untreated although effective treatments exist) for panic disorder is $55.9 \%$; for GAD is $57.5 \%$ and for OCD is $57.3 \% .^{5}$ In the UK, anxiety of all types is undertreated with $57 \%$ of adults with phobia in receipt of treatment, around $35 \%$ of those with GAD, and only $15 \%$ of those with mixed anxiety and depressive disorder. ${ }^{4}$ 
Depression is also under-recognised and underdiagnosed in general practice with approximately half of patients receiving a diagnosis. ${ }^{6-8}$

In the UK, general practice (GP) patient records have been used to understand prevalence and treatment of common mental health problems. ${ }^{9-11}$ Recognition of mental health problems in primary care only comes after the patient seeks medical care and discloses relevant symptoms, and the GP identifies and acknowledges the problem's psychological nature. Determinants of whether the GP will recognise psychiatric disorders include the way the patient describes their symptoms, biases held by the physician ${ }^{12}$ as well as time pressures on the physician. These steps are important because $90 \%$ of identified mental health problems are managed in general practice in the UK, particularly depression and anxiety. ${ }^{13}$ The monitoring and management of depression is now financially incentivised in UK general practice through the Quality and Outcomes Framework (QOF). ${ }^{14}$ The way GPs record depression and its treatment has become more standardised, and has been investigated in previous studies. ${ }^{15-18}$

Conversely, anxiety disorders are not covered by the financial incentives of QOF and there is no standardised way of recording a suspicion or diagnosis of anxiety. ${ }^{11}$ With multiple causes and manifestations, anxiety is often diagnosed only after excluding physical causes of the symptoms. This is considered necessary because patients with anxiety disorder commonly present in general practice with non-specific somatic symptoms. GPs report that although they recognise behavioural disturbances and distress, common presentations of symptom patterns and morbidity do not fit readily within the discrete diagnostic categories of anxiety disorders. ${ }^{7}$ GPs recording of patients' anxiety may be influenced by many factors, such as their own understanding or beliefs about anxiety, their (un)certainty of diagnosis, their ability to offer help or treatment, or the patient's own barriers or beliefs about anxiety as a disorder. ${ }^{11}$ They may also wish to wait to see if symptoms resolve over time or become a long-term issue for the patient. In the $50 \%$ of cases where anxiety symptoms are comorbid with depression, ${ }^{19}$ GPs may feel that a depression code is enough to capture the overall clinical picture. Some GPs also report that they feel they have fewer treatment options to offer patients with anxiety, which may disincentivise recording a diagnosis. Currently, using GP patient records to understand prevalence and treatment of anxiety is very problematic, especially as there has been a trend over the last decade towards GPs using symptom codes (eg, anxiousness-symptom; panic attack) and generic codes (eg, anxiety states) instead of specific diagnostic codes. ${ }^{11}$

GPs' diagnosis and recording of anxiety are not well explored in the literature, with few studies since the 1990s examining GPs' interactions with their coding systems. Given the widespread adoption of electronic medical records in British general practice since that time and their growing use for epidemiological research, it is important to explore coding behaviour once again. Studies from the 1990s may not be relevant to the current generation of GPs who interact with computer software in the knowledge that the records they create may be used for secondary purposes such as audit, service planning and research. In this study, we interviewed GPs directly with the aim of describing: (1) GPs' coding and recording of anxiety, and (2) the influences on their recording behaviours. We conducted a qualitative interview study asking GPs about their conceptualisation of anxiety, their approach to diagnosis, how they record consultations with regard to anxiety and why they do it that way.

\section{METHODS}

\section{Ethical approval}

Research and development approval was given by Sussex National Health Service (NHS) Research Consortium.

\section{Study design and procedure}

Semistructured interviews were conducted with GPs by two female medical students (AC and DAC) between December 2013 and March 2014, either at the GP's surgery or in the medical school. Interviews were conducted in a closed room with no one else present. The interview started with reading two fictional vignettes (box 1), and questions expanded from discussion of these cases. Vignettes were developed from text books ${ }^{20}$ and online resources ${ }^{21}$ and were piloted with two practising GPs. The questions initially focused on how participants would talk to and diagnose the patients in the

\section{Box 1 Case studies}

Sally is a 39-year-old divorced mother of two children. She was divorced a year ago after her husband, who had had a string of extramarital affairs, decided to leave her for another woman he had met at work. Soon after the divorce Sally took a job in a call centre in order to make ends meet. She has started having a lot of headaches. She has been having difficulty getting off to sleep for the past 6 months, is irritable, on edge and finds herself shouting at the children frequently. She has recently started experiencing palpitations and a tingling sensation in her hands. She spends most of the day worrying about various things, such as whether she is bringing up her children well, whether she will find another partner and whether she will get 'the sack'.

Andrew, a 26-year-old, is unemployed and afraid to leave his house. His fear of leaving the house started about a year ago when he was in the supermarket and suddenly experienced a feeling of sheer terror. His heart pounded he trembled; his mouth got dry and it felt as if the walls were caving in. He felt like he was totally out of control and might die. He had two subsequent attacks, both when he was out of his house, and since then he has been afraid to go out. On the occasions when he leaves his house, he insists that a friend accompany him and stay by his side until he returns home. 


\section{Box 2 Examples of interview questions}

Question (1) "What is your understanding of anxiety?"

Question (2) In relation to the case studies: "What would you document as your initial impression?"

Question (3) "If you would use a code, which codes would you be likely to use?"

Question (4) "How would you record different diagnoses of anxiety disorders? What would you code/write in the notes?"

Question (5) "Would you use a code relating to anxiety or a generic code plus free text? What would you write in the notes?"

Question (6) "What external influences are there on your choices of codes/text to record?"

vignettes, GPs' own perceptions of anxiety disorders, and how they would manage and record consultations with similar patients (box 2).

Interviewers received training to ensure uniformity of interview styles, and used a standardised interview schedule with a mixture of open and closed questions to elicit both specific answers and encourage free-flowing conversation. Interviews lasted an average of $24 \mathrm{~min}$ (SD $10 \mathrm{~min}$ ) and were audio-recorded; no notes were made.

\section{Read codes}

After reading the vignettes, GPs were asked how they would record the consultation with the patient, using the coding system specific to UK general practice, called Read codes. Read codes are a hierarchically structured vocabulary developed by a UK GP in the 1980s, called Dr James Read. They map to other nomenclatures such as International Classification of Disease codes and International Classification of Primary Care codes. Each Read code represents a term or short phrase describing health-related concepts such as diagnoses, symptoms, tests, referrals, administration and correspondence. There are over 200000 different codes, which are sorted into categories (diagnoses, processes of care and medication) and subchapters. ${ }^{22}$ Each clinical entity is represented by a 5 byte alphanumeric code and a Read term which is the plain language description. The way that GPs use Read codes varies, but many describe choosing a 'summary' code which is a keyword representing the main body of the consultation. ${ }^{23}$ The GP may then add text beside the code to capture complexity, evolving circumstances, uncertainty and severity. ${ }^{24}$

\section{Participant recruitment}

A convenience sample of currently practising GPs was recruited face to face, and through email adverts, through networks of contacts in a medical school in the South East of England. GPs expressing an interest were sent information leaflets about the study and gave written consent when they agreed to participate. As the study was advertised widely, it was not possible to calculate refusal rates. Recruitment ceased when there was consensus that data saturation had occurred (between
$\mathrm{AC}, \mathrm{DAC}, \mathrm{MC}$ and $\mathrm{EF})$. Interviews were transcribed and coded immediately, in parallel with subsequent interviews, and by the 16th and 17 th interview, it was noted that no new themes were emerging.

\section{Analysis}

The interview transcripts were analysed thematically, ${ }^{25}$ using an inductive approach which focused on creating themes directed by the content of the data. This approach was advantageous because of its flexibility in methods of interpretation, but limited in the sense that it only allowed for a largely descriptive summary of themes. ${ }^{26}$ We were guided by a subtle realist-interpretivist position, striving to be as neutral and objective as possible in the collection, interpretation and presentation of the data. ${ }^{27}$ Initial identification of themes across the transcripts was carried out, and in an iterative process, codes were generated that arranged features into groups of meaningful concepts using NVivo software (by HH-B, MC, EF). The transcripts were studied again to explore dimensions of these concepts and the system thus refined. Each theme is presented using key illustrative quotations. A summary of findings was sent to all participants.

\section{RESULTS}

Seventeen GPs were recruited and participated in this study (table 1).

\section{Choice of codes}

In relation to documenting the two vignettes, GPs were asked 'Which codes would you be likely to use?'. The range of Read codes stated by the GPs are summarised for each vignette in table 2. GPs chose a range of Read codes, some of which were only loosely related to anxiety, while others were quite specific. Of the 17 participants, 12 mentioned they would use free text in the recording of anxiety, 9 described what they would document in the free text although 3 GPs said they would just write "what's going on", "what the patient exactly said" or "what I am worried about". Six GPs stated

\begin{tabular}{ll} 
Table 1 Participant information & \\
\hline Gender & 9 female, 8 male \\
Part-time or full-time work & 9 part time, 8 full time \\
Age range & $31-40$ years 4 GPs \\
& $41-50$ years 6 GPs \\
& $51-60$ years 7 GPs \\
& 14 (range 1-30) \\
Average number years & 11 South East England \\
in practice & 3 North Wales \\
Location of practice & 3 West Midlands \\
& 9250 patients \\
Average practice size & (range 5350-16 000) \\
\hline GP, general practitioner. &
\end{tabular}


Table 2 Read codes chosen by general practitioners (GPs) for each vignette

\begin{tabular}{lll}
\hline Read term & $\begin{array}{l}\text { Vignette: Sally } \\
\text { (number of GPs } \\
\text { giving code) }\end{array}$ & $\begin{array}{l}\text { Vignette: Andrew } \\
\text { (number of GPs } \\
\text { giving code) }\end{array}$ \\
\hline Anxiety & 7 & 5 \\
Anxiety states & 0 & 3 \\
Anxiety attacks & 2 & 1 \\
Anxiousness & 1 & 1 \\
symptom & & \\
Generalised & 3 & 1 \\
anxiety & & \\
Anxiety and/with & 7 & 2 \\
depression & & 1 \\
$\begin{array}{l}\text { Depression } \\
\text { Stress-related }\end{array}$ & 2 & 0 \\
problem & 2 & \\
Stress & 1 & 0 \\
$\begin{array}{l}\text { Panic attack } \\
\text { Panic disorder }\end{array}$ & 0 & 5 \\
Agoraphobia & 0 & 1 \\
\hline $\begin{array}{l}\text { NB, participants could respond with more than one code per } \\
\text { vignette. }\end{array}$ & \\
\end{tabular}

definitively what aspects they would document: history $(10,12,17)$ symptoms $(2,3,8,10)$ assessment/examination $(12,17)$, discussion of management plan $(3,10$, 17), social context (3) and Hospital Anxiety and Depression Scale (HADS) score (3). One participant said explicitly "well we don't use free text very much because nobody reads it...basically" (15) perhaps reflecting this participant's experience working as a GP in a hospital emergency medicine department where there is a lack of continuity between clinicians and patients.

\section{Coding culture-influences on how anxiety is documented} Seven themes arose from the data that represent influences on GP's recording, and which reflect a wider 'coding culture', within the specific exemplar of anxiety.

\section{Theme 1: anxiety-or a normal response to stress?}

Almost all participants responded that they felt confident in recognising symptoms of anxiety, particularly physical ones. However, many clinicians noted that it was difficult with some patients to distinguish anxiety that was a 'normal' response to stress from more serious or chronic presentations that interfered with everyday life and required more detailed documentation and management. In response to the former, participants either avoided applying an anxiety code or resorted to using broad Read codes such as 'stress at home'. Behind this was a widespread desire to avoid medicalising anxiety that was just a 'natural' response to stressful life events:

I don't want to sort of start 'medicalising' her because as

far as I'm concerned there's a lot of life events, this is life

-we have to deal with it! (5).
They also considered that anxiety was a normal part of individual's lives and would only choose to diagnose it when it became 'debilitating' (2).

It's a spectrum, it's a degree so it often is a kind of decision as to how much it's affecting that person's life which then determines whether you call it anxiety. (2)

\section{Theme 2: granularity of diagnosis—getting it 'good enough'} Participants gave the sense that reaching the exact diagnosis was not as important as getting the right management plan in place. The same strategies were used for recognising anxiety as for any other mental health diagnosis, for example, visual cues from the patient:

Central to this what you don't really get with this case study is that you can't just look at the patient and I think with depression you often do get clues as to whether it is. (11)

GPs did not attempt to differentiate between different types of anxiety such as 'depression with anxiety, GAD, anxious symptoms, panic attacks' (13), and doubted their competence to code such a detailed diagnosis:

Whether I would be happy, have the balls, to write, code it as obsessive compulsive disorder or whether I would fob it off as depression, I'm not sure...(13)

Instead they just aimed to 'document what was going on' (1) in a general code:

The big two codes that we use mainly for mental health, one is anxiety, one is depression. And that's it. We're simple people. (5)

Participants in this study questioned the utility and diagnostic validity of the wide selection of available Read codes for anxiety. This was because of 'grey areas' (13) that could result where symptoms overlapped, fluctuated or a patient had coexisting conditions such as anxiety and depression. GPs overall aim was to develop a suitable management plan for the particular patient, with or without a specific diagnosis. Despite sometimes feeling 'out of their depth' (13), this approach appeared to be effective:

Patients clearly like what I'm telling them because they're coming back and seeing me and they've got trust in me, but I feel quite uncomfortable with the fact that I'm just sort of following my nose...I'm not really sure I have confidence in what I'm doing. (13)

\section{Theme 3: giving patients a 'label'-worry about stigma}

The majority of GPs stated they would be reluctant to code a patient with an anxiety disorder at their first consultation. This was from a desire to avoid prematurely coding anxiety, partly because of diagnostic uncertainty, but also due to the perception that such a code would be 'stigmatising' (14). 
You don't immediately stick a label on them as being anxious. (14)

Practitioners also expressed concern about the permanence of patients' medical records and urged caution about making an entry in haste:

GPs can get a little bit ahead of themselves and start labelling patients with something...it's very difficult to get rid of that label. (13)

Other participants were concerned with the permanence of such a Read code for specific practical considerations, for example, the implications for future insurance:

That (coding) I might be a bit more canny about... because I think there are potential implications when someone's applying for a mortgage or insurance, to have a hard Read coded diagnosis. (12)

Some clinicians would avoid formally recording an anxiety disorder due to pressure from patients, who did not accept their diagnosis or questioned its validity:

Sometimes the patient is uneasy with certain diagnoses and sometimes they tell you that. That can be an external factor...(to coding). (11)

Some clinicians even reported documenting anxiety with Read codes that were totally non-specific and which added little to the value of data entry: "I often put 'seen in GP's surgery' if I'm going to do a generic code.” (16).

\section{Theme 4: time as a tool-'next week they'll be fine'}

Coding was described by some as being a fluid process, evolving and developing over a number of consultations as the diagnosis was refined.

I'd probably just put down at this stage as a stress related problem...the diagnosis of anxiety would come not with just one interview but with a series of interviews. (2)

This strategy reflected the sometimes ambiguous nature of psychiatric diagnoses, due to fluctuating or overlapping symptoms, uncertainty at what was 'pathological' versus 'normal' worry and the GP's experience that symptoms could spontaneously resolve over time.

If I was to use a code...urm, the first time you ever see someone you don't necessarily [enter a Read code] because you might see them next week and say 'oh it's fine' which just happens so often. (16)

With this perspective in mind, GPs suggested they would follow-up the patient: "you'd be reviewing them again you see" (16) and factor time into the management plan as an aid to resolution of symptoms:

Then we could just give her a bit of time to think or talk to certain people or change a few bits basic stuffs in her life, and just get her back, you know a lot of stuff eases off after time. (5)

\section{Theme 5: justifying the choice of code}

A number of practitioners expressed doubts about their Read-coding abilities: "I'm not good in coding" (1). Some lacked confidence generally in being able to translate a clinical diagnosis to a Read code, while others experienced difficulty because of the perception that there were too many codes to choose from.

But I don't know how you do it (coding) well...you know, how do you choose that code? (12)

This led to some participants either not coding at all, and only using free text to document consultations and diagnoses; or using one of three strategies for justifying the code chosen, all of which drew on other sources of information:

First was to use a formal screening tool (such as GAD 7 ) as 'evidence' and 'as the main factor in determining what to code' (17).

The second strategy for choosing codes was to defer to mental health professionals by "wait(ing) for the psychs or psychologists to give...the proper Read codes" (12).

Third, in the absence of these influences, GPs tried to standardise coding between doctors in their clinics stating that their strategy was to look at 'what did the doctor before you used and copy that' (13).

'Copying' the codes and aiming for consistency between practitioners could however lead to the use of more general codes:

We tend to keep it general, quite general because then we've got more chance [that] most people in surgery will code it similar and you'll find it if you need to search for it... (5)

This strategy was perceived to be helpful in aiding consistency of care and information retrieval:

If you choose a code, how do you know that everyone else in the organisation is going to do it...It's an absolute nightmare and it matters when you want to retrieve information.(12)

Some GPs additionally described that they would be told what terms to use in practice meetings to ensure external services could be accessed patients:

The only thing that would affect me...is if in maybe one of the staff meetings, someone said 'oh there's a new support group opening up or something but in order to access it you need to label the patient as this or you need to put this in a referral or a dictation'. (13)

\section{Theme 6: perceptions about usefulness of coding in general}

There were differences of opinion about the usefulness of coding in contributing to patient care. Some clinicians questioned the necessity of having a Read code 
system as they believed it did not affect patient management:

But I'm not sure it (coding) particularly brings anything more to the party...I'm not sure how useful it is to have a strict coding system. (11)

(coding) on a practical basis it's irrelevant really....(13)

Conversely, others believed that in certain cases it could be beneficial, for example, where there was a clear treatment protocol for a diagnosis. A number of participants believed coding was useful for 'statistical purposes' (17) and resource allocation both at a national level, and in terms of service provision within individual surgeries.

Some practitioners were of the view that the coding process was useful in 'putting a name' (5) to what patients' were experiencing, and that it could 'empower' (5) patients, such that they could start to take their problem forward:

I guess to the patient it might be quite useful to have it kind of categorised. (8)

\section{Theme 7: practice-specific pressures}

A factor identified by a number of clinicians that influenced coding behaviour was time pressure. Many participants felt that they did not have enough time to find the most appropriate Read code and that 'it could take you 10 minutes to find the right code' (16). One reason for this was because some GPs believed it was more important to dedicate all the available consultation time to the patient.

I'm probably more guilty of putting more time into the discussion than the recording of the discussion. (11)

Some GPs reported that practices had tried to address wider time pressures by employing non-medical staff to code. There were differing opinions as to the effectiveness of this with some finding it useful: “....and she'll pick up the right code which is lovely" (12), while others expressed concern about non-clinical staff interpreting and transcribing data from consultations.

Another factor identified by a minority of clinicians was the influence of coding software on inputting Read codes, with codes that were selected most frequently being more prominent and more likely to be used.

Only I suppose it's governed by what codes are prominent on our IT system. (10)

Finally, the exclusion of anxiety from the QOF meant that some practitioners felt they experienced less pressure to diagnose anxiety than other mental health conditions, in particular depression.

If you diagnose someone as being depressed you know you've got a hell of a lot of boxes to tick on a regular basis...so there's actually less pressure on anxiety...so we've got some benefit to diagnose someone as anxious rather than depressed. (5)

\section{DISCUSSION}

This study identified multiple dimensions of a 'coding culture' in general practice that emerged from investigating the exemplar condition of anxiety. Influences on coding included recognition of anxiety as a normal state which may resolve over time. This knowledge led to uncertainty over diagnosis in initial consultations, and coupled with the perceived stigma of having a permanent label, it shifted the chosen Read codes towards more symptom-based ones. Alternatively, non-specific or administrative codes were entered and symptoms and history documented in the free text.

The vignettes we used were static and only represented a single consultation. In response to this stimulus, where information was somewhat ambiguous and no questions could be asked of the patient, 12 of 17 GPs said they would use descriptive free text to supplement coded information. A wide variety of recording styles was evident, as in relation to the two vignettes the 17 participants chose 12 different codes ranging from the vague 'stress' to the more specific 'agoraphobia'. When choosing a code, GPs sought to have justification for the code chosen, such as test scores, letters from specialist and harmonising codes between practitioners in their clinic. In addition they reported accepting suggestions made by their coding software in order to save time.

Owing to the ambiguity of initial presentations of anxiety, GPs suggested that they used time as a tool in two ways. First to increase certainty over the diagnosis, and second as a form of management, as anxiety could get better over time even without clinical intervention. This suggests a pragmatic attitude to resolving both clinical uncertainty and to dealing with constraints on resources by adopting a wait and see approach, and to enable a relationship of trust to develop between doctor and patient. ${ }^{28}$ Watchful waiting is a recommended approach for other mild mental health conditions such as depression. ${ }^{29}$ This approach was also evident in their management plan which was usually to 'bring the patient back' to see them within a short time frame. Interestingly, time was also seen as a constraint to good coding within patient consultations, as GPs said they had to choose between focusing on the patient, or focusing on recording the discussion.

This study additionally reveals a tension between a static coding system and the way mental health is managed in general practice. There is a wider difficulty exposed here in categorising mental health problemsthe classification of which is continually discussed and adjusted (eg, in Diagnostic and Statistical Manual of Mental Disorders, Fifth Edition (DSM-V) ${ }^{30}$ ). Psychiatric diagnoses lack consensus on their validity even in specialist settings, and in primary care, many patients present with clear distress but with undifferentiated symptoms 
which may fluctuate over time, rather than a discernible disorder fitting a psychiatric category. ${ }^{31}$ Previous research on depression suggests that primary care physicians hold two conflicting models of depression, a biomedical understanding, supplemented by a recognition of the psychosocial context of depression. These arise due to their biomedically oriented training, coupled with their everyday experiences and awareness of patients' daily lives. ${ }^{32}$ This can lead to apparent dissonance or tension in the way GPs approach depression, and this may hold true for anxiety. In mental health consultations, GPs have several goals to achieve. They must exclude a physical cause for the problem before settling on a psychological explanation and work within the wider context of the patient's social environment, current stressors and other illnesses, without overpathologising normal responses to those stressors. Evidence reported here suggests that it is likely that the GP aims to get the diagnosis to only the level of granularity at which an appropriate and feasible management plan can be implemented.

Additionally, GPs perceive negative consequences for the patient of having a mental health diagnosis recorded. In our study, GPs referred to implications for applying for a mortgage or for insurance, and this is borne out by other studies. For example, Rost $e t a l^{33}$ reported that over $50 \%$ of US-based primary care physicians had deliberately coded depression as something else in a 2-week period, for reasons of uncertainty or problems with reimbursement for the patient. The most common substitutions were fatigue/malaise and insomnia. Reimbursement is not an issue for the patient in the UK, but there still appears to be a hesitation to formally label a patient when any uncertainty exists.

Our findings are consistent with Walters $e t a l,{ }^{11}$ who found that the recording of anxiety symptoms rather than firmer diagnoses was increasing in recent years. Like us, they speculate that this might be because of an increasing debate over the meaning and value of discrete psychiatric categories, in particular for patients with milder presentations. Walters et $a l^{11}$ also conjecture that GPs may be uncertain of or lack training in the criteria needed for firm diagnoses, that they may believe that distinctions are not meaningful in primary care practice and that they are reluctant to stigmatise patients. We have been able to show that labels are a genuine concern for GPs, and that they are unwilling to firmly code anxiety disorders without additional evidence for the diagnosis.

\section{Implications for future research}

With the numerous influences reported on recording practices, it remains a difficult task to predict how anxiety cases may best be ascertained from patient records for research and audit purposes. By acknowledging the existence of a wider coding culture, researchers should be aware that GPs use symptom and other nonspecific codes in their records and that making and coding a firm psychiatric diagnosis may be less of a priority than formulating an appropriate management plan. The variety of strategies for documenting anxiety presents a problem for researchers ascertaining cases. It is clear that both high order diagnostic codes and symptom codes should be included in case ascertainment strategies and that to increase sensitivity, free text should also be considered. Owing to codes evolving from more vague to more precise within the patient record, case ascertainment could also usefully have a time element incorporated.

Of interest was the fact that GPs tried to harmonise coding at a practice level, suggesting that codes for anxiety may be standardised within a practice but not between practices. Electronic health records (EHR) researchers may therefore wish to factor practice-level effects into their case ascertainment strategies. Currently the curriculum of the Royal College of General Practitioners does not include specific Read code training, ${ }^{34}$ so it is not clear how individuals or practices develop their coding strategies.

\section{Strengths and limitations}

This is the first UK study looking at influences on GPs' coding behaviour with regard to anxiety. This is an important condition and one that GPs may approach differently from other common mental health problems due to its overlap with somatic symptoms, and the lack of financial incentive for its diagnosis and management. However, this is a small qualitative study, and therefore it is not known if the results can be generalised across the UK population of GPs. Certainly, results are unlikely to generalise to other countries' primary care systems, especially those which do not use Read codes, or where mental health is managed in specialist settings. A further potential weakness was that this study was undertaken by a team of researchers rather than in-depth by one researcher. On the other hand, this approach offers insight into diverse representations of the phenomenon under study, thus potentially strengthening the findings of the study. ${ }^{35}$

An additional limitation is the approach of using static vignettes, whereas in real life, the GP would have the opportunity to invite the patient back and observe how their condition develops over time. However, increasingly, British GPs are working in larger surgeries without a named doctor-patient relationship and personal knowledge of patients, and therefore may have to make assessments about mental health the first time they meet the patient or on the basis of notes made by colleagues. It is clear that it may not be clinically appropriate to give a firm diagnosis on the first meeting, but this study still illustrates the wide variation in approach to recording, highlighting the problems for EHR researchers.

\section{CONCLUSIONS}

This study has identified dimensions of a coding culture in general practice that appear to arise from clinical uncertainty, a long-term perspective and a focus on clinical management rather than diagnosis. The coding 
strategies described reflect core clinical challenges facing generalists working in the community. For that reason, it is unlikely that coding training or more userfriendly software will improve the epidemiological usefulness of clinical codes for mental health in general practice. Greater research attention should therefore be paid to the free-text records made by GPs, especially for conditions like anxiety that can present with 'normal' symptoms, be stigmatising or impact on insurance.

Acknowledgements The authors wish to thank the GPs who gave their time to participate in this study.

Contributors EF conceived and designed the study. AC and DAC were involved in data collection. $\mathrm{HH}-\mathrm{B}, \mathrm{MC}$ and $\mathrm{EF}$ were involved in data analysis. $\mathrm{EF}, \mathrm{MC}$ and $\mathrm{HH}-\mathrm{B}$ were involved in writing the manuscript. All authors read and approved the final version.

Funding This research received no specific grant from any funding agency in the public, commercial or not-for-profit sectors.

Competing interests None declared.

Ethics approval Brighton and Sussex Medical School Research Governance \& Ethics Committee

Provenance and peer review Not commissioned; externally peer reviewed.

Data sharing statement Additional data in the form of anonymised typewritten interview transcripts are available by emailing e.m.ford@bsms.ac.uk

Open Access This is an Open Access article distributed in accordance with the Creative Commons Attribution Non Commercial (CC BY-NC 4.0) license, which permits others to distribute, remix, adapt, build upon this work noncommercially, and license their derivative works on different terms, provided the original work is properly cited and the use is non-commercial. See: http:// creativecommons.org/licenses/by-nc/4.0/

\section{REFERENCES}

1. Prince M, Patel V, Saxena S, et al. No health without mental health Lancet 2007;370:859-77.

2. Whiteford HA, Degenhardt L, Rehm J, et al. Global burden of disease attributable to mental and substance use disorders: findings from the Global Burden of Disease Study 2010. Lancet 2013;382:1575-86.

3. Somers JM, Goldner EM, Waraich P, et al. Prevalence and incidence studies of anxiety disorders: a systematic review of the literature. Can J Psychiatry 2006;51:100-13.

4. McManus S, Meltzer H, Brugha T, et al. Adult psychiatric morbidity in England, 2007: results of a household survey. London, UK: The NHS Information Centre for Health and Social Care, 2009.

5. Kohn R, Saxena S, Levav I, et al. The treatment gap in mental health care. Bull World Health Organ 2004;82:858-66.

6. Mitchell AJ, Vaze A, Rao S. Clinical diagnosis of depression in primary care: a meta-analysis. Lancet 2009;374:609-19.

7. Hickie IB. Primary care psychiatry is not specialist psychiatry in general practice. Med J Aust 1999;170:171-2.

8. Verhaak PF, Schellevis FG, Nuijen J, et al. Patients with a psychiatric disorder in general practice: determinants of general practitioners' psychological diagnosis. Gen Hosp Psychiatry 2006;28:125-32

9. Rait G, Walters K, Griffin M, et al. Recent trends in the incidence of recorded depression and depressive symptoms in primary care. Br J Psych 2009;195:520-4.

10. Bhattarai N, Charlton J, Rudisill C, et al. Prevalence of depression and utilization of health care in single and multiple morbidity: a population-based cohort study. Psychol Med 2013;43:1423-31.
11. Walters K, Rait G, Griffin M, et al. Recent trends in the incidence of anxiety diagnoses and symptoms in primary care. PLOS ONE 2012;7:e41670.

12. Goldberg D, Huxley P. Mental illness in the community: the pathway to psychiatric care. London: Tavistock Publications, 1980.

13. Royal College of General Practitioners. The RCGP Curriculum: Clinical Modules 3.10 Care of People with Mental Health Problems. 2015 (cited 06/10/15). http://www.rcgp.org.uk/ /media/Files/ GP-training-and-exams/Curriculum-2012/RCGP-Curriculum-3-10Mental-Health-Problems.ashx

14. NHS England. Quality and Outcomes Framework guidance for GMS contract 2013/14. 2013. https://www.google.co.uk/url?url=https:// www.bma.org.uk/-/media/Files/PDFs/Practical\%2520advice\%2520at $\% 2520$ work/Contracts/gpqofguidance20132014.pdf\&rct=j\&frm= $1 \& q=\& e s r c=s \& s a=U \& v e d=0 a h U K E w j z v c v V 8 b T N A h X I P x Q K H S-$ QA4EQFggUMAA\&usg=AFQjCNHZFex-JkKfSaOg4jtf38iz4SEJxA

15. McCall L, Clarke D, Trauer T, et al. Predictors of accuracy of recognition of emotional distress in general practice. Prim Care Community Psychiatr 2007;12:1-5.

16. van Rijswijk $\mathrm{E}$, van Hout $\mathrm{H}$, van de Lisdonk $\mathrm{E}$, et al. Barriers in recognising, diagnosing and managing depressive and anxiety disorders as experienced by family physicians; a focus group study. BMC Fam Pract 2009;10:52.

17. Hyde J, Calnan M, Prior L, et al. A qualitative study exploring how GPs decide to prescribe antidepressants. Br J Gen Pract 2005;55:755-62.

18. Mitchell C, Dwyer R, Hagan T, et al. Impact of the QOF and the NICE guideline in the diagnosis and management of depression: a qualitative study. Br J Gen Pract 2011;61:e279-89.

19. Fava M, Rankin MA, Wright EC, et al. Anxiety disorders in major depression. Compr Psychiatry 2000;41:97-102.

20. Ayers S, De Visser R. Psychology for medicine. Sage, 2010.

21. National Institute for Health and Care Excellence (NICE). Generalised anxiety disorder and panic disorder (with or without agoraphobia) in adults. Clinical case scenarios for generalised anxiety disorder for use in primary care. 2011. https://www.nice.org. uk/guidance/cg113/resources/clinical-case-scenarios-pdf-136292509

22. Chisholm J. The Read clinical classification. BMJ 1990;300:1092

23. Ford E, Nicholson A, Koeling R, et al. Optimising the use of electronic health records to estimate the incidence of rheumatoid arthritis in primary care: What information is hidden in free text? BMC Med Res Methodol 2013;13:105.

24. de Lusignan S, Wells SE, Hague NJ, et al. Managers see the problems associated with coding clinical data as a technical issue whilst clinicians also see cultural barriers. Methods Inf Med 2003;42:416-22.

25. Guest G, MacQueen KM, Namey EE. Applied thematic analysis. Sage, 2011.

26. Braun V, Clarke V. Using thematic analysis in psychology. Qual Res Psychol 2006;3:77-101.

27. Ritchie J, Lewis J, Nicholls CM, et al. Qualitative research practice: a guide for social science students and researchers. Sage, 2013.

28. Heneghan C, Glasziou $\mathrm{P}$, Thompson $\mathrm{M}$, et al. Diagnostic strategies used in primary care. BMJ 2009;338:b946.

29. National Institute for Health and Care Excellence (NICE). Clinical Guideline 23 Depression: management of depression in primary and secondary care. 2004. https://www.nice.org.uk/guidance/CG023

30. American Psychiatric Association p. Diagnostic and statistical manual of mental disorders: DSM-5. 5th edn. Arlington, VA: American Psychiatric Association, 2013.

31. Gask L, Klinkman M, Fortes S, et al. Capturing complexity: the case for a new classification system for mental disorders in primary care. Eur Psychiatr 2008;23:469-76.

32. Thomas-MacLean R, Stoppard JM. Physicians' constructions of depression: inside/outside the boundaries of medicalization. Health 2004;8:275-93.

33. Rost K, Smith GR, Matthews DB, et al. The deliberate misdiagnosis of major depression in primary care. Arch Fam Med 1994;3:333.

34. Royal College of General Practitioners. Information Management and Technology; Curriculum Statement 4.2. 2007 (cited 23rd February 2016). http://www.rcgp.org.uk/training-exams/ gp-curriculum-overview/ /media/Files/GP-training-and-exams/ Curriculum-previous-versions-at-July-2012/RCGP-Curriculum-4-2IMT-2009.ashx

35. Malterud K. Qualitative research: standards, challenges, and guidelines. Lancet 2001;358:483-8. 